\title{
DOCTRINA
}

\section{La educación ambiental en la legislación de Argentina}

\author{
Environmental education in Argentine legislation
}

\begin{abstract}
Verónica Lucía Cáceres (iD)
Universidad Nacional de General Sarmiento, Consejo Nacional de Investigaciones Científicas y Técnicas, Universidad Nacional de Luján, Argentina
\end{abstract}

RESUMEN La preocupación por la situación del ambiente es un problema global que involucra derechos humanos inalienables de creciente importancia para la sostenibilidad de la vida. Este artículo tiene como objetivo analizar el lugar que asume la educación ambiental formal en el sistema educativo obligatorio de la República Argentina. En ese sentido, entiende que la educación ambiental conforma un instrumento de política y gestión de tipo preventivo que contribuye a propiciar conductas y valores relacionados con la protección del ambiente. Se trata de un trabajo exploratorio con una metodología cualitativa que recurre al análisis documental. A lo largo del trabajo se conceptualizan algunos aspectos de la educación ambiental como herramienta de gestión preventiva y se estudia el ordenamiento jurídico que la sostiene a nivel nacional y provincial. Las conclusiones permiten observar que la educación ambiental formal ha permanecido más allá de los cambios en las políticas ambientales y se ha institucionalizado en un conjunto de normativas altamente fragmentadas.

PALABRAS CLAVE Educación ambiental, federalismo, desarrollo sostenible, gestión ambiental preventiva.

ABSTRACT Concern for the state of the environment is a global problem that involves inalienable human rights of growing importance for the sustainability of life. The purpose of this article is to analyze the place of formal environmental education in the compulsory education system of the Argentine Republic. It also understands that environmental education is a preventive policy and management instrument that contributes to promoting social behavior and values related to environmental protection. It is an exploratory work with a qualitative methodology that is based on documental analysis. Throughout this work some aspects of environmental education are conceptualized; the process of institutionalization at a national and provincial levels is looked through; and the common curricula contents, related to the environment established for the educa- 
tional system are presented. Conclusions allow us to observe that formal environmental education has remained, beyond the changes in environmental policies and the organization of the State, and a set of regulations, organisms, and curricular contents in the school that had been institutionalized.

KEYWORDS Environmental education, federalism, sustainable development, preventive environmental management.

\section{Introducción}

La preocupación por la situación del ambiente involucra derechos humanos inalienables de creciente importancia para la sostenibilidad de la vida. La crisis ecológica profunda que afecta al aire, los suelos, los recursos hídricos, la flora y la fauna se cristaliza en los territorios incrementando las desigualdades sociales y económicas. En la agenda internacional, la crisis ambiental adquirió mayor protagonismo en la década de los setenta del siglo XX y se consolidó en el marco de las conferencias de la ONU conocidas como las Cumbres de la Tierra. Desde entonces se han abordado y problematizado distintas herramientas de gestión ambiental de tipo preventivas ( $\mathrm{y}$ también correctivas), y la educación ambiental es una de ellas.

La educación ambiental conforma una herramienta preventiva que se ha institucionalizado con distinto grado y nivel en el sistema educativo de la región, incluyendo la República Argentina, aunque sigue conformando un espacio en construcción. Principalmente, la educación ambiental surgió a partir de la conferencia de la ONU de Estocolmo de 1972, que en su principio 19 destacó que la educación en cuestiones ambientales era indispensable para «ensanchar las bases de una opinión pública bien informada y de una conducta de los individuos, de las empresas y de las colectividades inspirada en el sentido de su responsabilidad en cuanto a la protección y mejoramiento del medio en toda su dimensión humana» (ONU, 1973.

La República Argentina es un país federal donde la educación inicial, primaria, secundaria y superior no universitaria conforman una responsabilidad de los niveles subnacionales de gobierno: las provincias. El sistema educativo obligatorio comienza en el nivel inicial con la sala de cuatro años hasta la escuela de educación secundaria. El nivel superior tiene una amplia oferta educativa integrada por las universidades, los institutos universitarios y los institutos de educación superior. Dado lo anterior, las normativas educativas entrelazan disposiciones nacionales y provinciales y se diseñan lineamientos generales en instancias federales de coordinación.

Por otro lado, en materia ambiental, la reforma de la Constitución Nacional de 1994 estableció que las provincias le delegan a la nación la potestad de dictar normas en materia de presupuestos mínimos de protección ambiental. Así lo establece el ar- 
tículo 41 que sostiene: «Corresponde a la Nación dictar las normas que contengan los presupuestos mínimos de protección, y a las provincias, las necesarias para complementarlas, sin que aquellas alteren las jurisdicciones locales».

La reglamentación del artículo 41 dio lugar a la sanción de distintas leyes de presupuestos mínimos de protección ambiental, como la Ley 25.675 General del Ambiente de 2002. El artículo 14 de esta norma estableció a la educación ambiental como un «instrumento básico para generar en los ciudadanos, valores, comportamientos y actitudes que sean acordes con un ambiente equilibrado, propendan a la preservación de los recursos naturales y su utilización sostenible, y mejoren la calidad de vida de la población». También acordó que era función del Consejo Federal de Ambiente (Cofema) implementar planes y programas de educación ambiental para todo el territorio.

En 2006 se sancionó la Ley 26.206 de Educación Nacional, un hito en materia educativa por los cambios que introdujo en la organización y el reconocimiento explícito de la educación como derecho y la obligatoriedad de los estudios secundarios. En materia ambiental dicha norma sostuvo que el Consejo Federal de Educación (CFE) era el espacio de debate de la política educativa en general y acordó las medidas necesarias para proveer educación ambiental, en coordinación con el Cofema. Por lo que desde entonces la educación ambiental tiene un claro reconocimiento que demanda la articulación de dos instancias de organización federal.

Este artículo estudia, precisamente, la institucionalidad que sostiene la educación ambiental en la educación formal obligatoria en la República Argentina y el ordenamiento jurídico en el que se incorpora. Al estar la educación formal obligatoria descentralizada y conformar una responsabilidad de las jurisdicciones provinciales, cada una de ellas ha sancionado distintas normativas que incorporan, de forma diversa, la educación ambiental. Así, se argumenta que este tipo de educación, como parte del campo de debate ambiental, se estableció ya en la década de los noventa del siglo $\mathrm{XX}$ siguiendo la tendencia internacional y que, aún, con la sanción de la Ley 26.206 de Educación, se sostiene en un entramado normativo fuertemente atomizado que diluye su implementación efectiva. En ese marco se plantea que la reciente sanción de la Ley 27.621 de Educación Ambiental Integral, en 2021, configura una oportunidad para transformar esta situación y garantizar su incorporación de forma integral. El trabajo permite observar la trayectoria institucional que devino, finalmente, en la sanción de una Ley Nacional de Presupuestos Mínimos de educación ambiental.

A continuación, se da cuenta de la metodología utilizada, se enfatiza en la importancia de la educación ambiental como herramienta de tipo preventiva, y se caracteriza la situación jurídica de la educación ambiental en el territorio considerando sus núcleos centrales a nivel nacional y subnacional. 


\section{Metodología}

La educación ambiental en la República Argentina ha sido poco abordada en la literatura reciente, en especial desde la reforma educativa de 2006, por lo que este trabajo asume un carácter exploratorio. La aproximación metodológica es cualitativa y se basó en la observación y análisis documental de normativas (voluntarias y obligatorias).

Para la búsqueda y sistematización se utilizaron las siguientes bases especializadas: Información Legislativa y Documental, Normas de la Provincia de Buenos Aires, Normativa del Gobierno de Córdoba, y del Sistema de Información Normativa de la provincia de Santa $\mathrm{Fe}$, entre otras. Se consideraron también los proyectos de ley de educación ambiental mediante las bases públicas de la Cámara de Diputados de la Nación y la Cámara de Senadores de la Nación, las resoluciones y declaraciones del CFE y el Cofema. ${ }^{1}$

Se llevó adelante el análisis documental de 13 normativas nacionales, 27 provinciales y 14 proyectos de ley presentados por legisladores del oficialismo y la oposición. ${ }^{2}$ La delimitación temporal inició con la sanción de la Ley General del Ambiente en 2002 y se extendió hasta 2021 con la sanción de la Ley 27.621 de Educación Ambiental Integral.

1. Se consultaron de la Cámara de Diputados de la Nación los Expedientes 6755-D-2018, 6230-D2018, 279-D.-200, 2.771-D.-2006, 1.006-D.-2014, 1.314-D.-2014, 1.982-D.-2014, 2.090-D.-2014, 2.201-D.2015. Así de la Cámara de Senadores de la Nación se consultaron los expedientes 2437/2018, 3220/2015, 149/2015, 2086/2017, 2486/2019.

2. De la Provincia de Córdoba, la Ley de Política Ambiental 10.208/1; de San Juan, la Ley General del Ambiente Ley 513-L-2014 y Ley General del Ambiente Ley 513-L-2014; de Buenos Aires, la Ley de Educación 13.688; de Chubut, el Decreto 350; de Tierra del Fuego, la Ley Provincial Medio Ambiente 55/92; de Río Negro, la Ley de Educación Ambiental 4242/2007; de La Pampa, la Ley Ambiental de la provincia 1914; de Santa Cruz, la Ley Provincial de Educación 3305/12; de Misiones, la Ley XVI 80 de Educación Ambiental; de Jujuy, la Ley Provincial de Ambiente 5063; de Entre Ríos, la Ley de Educación Ambiental 10402/15; de Mendoza, la Ley 5.961/92 Preservación del Medio Ambiente y Ley 6.970/o2 de Educación Provincial; de Formosa, la Ley 1.06o Política Ecológica y Ambiental; de Chaco, las leyes de Educación Ambiental 1114-E, la Ley Provincial de Residuos Sólidos Urbanos 2028 R y la Ley Provincial de Recursos Naturales N 783; de Neuquén, la Ley Orgánica de Educación de Neuquén 2945/14; de La Rioja, la Ley provincial de Medio Ambiente de La Rioja 7801/05; de Santiago del Estero, la Ley de Educación 6876 y la Ley 6.321 de Defensa, Conservación y Mejoramiento del Ambiente y los Recursos Naturales; de la Ciudad de Buenos Aires, la Ley de Educación Ambiental 1687/05; de Corrientes, la Ley 6.265; de Catamarca la Ley 5.424 y de Tucumán la Ley 6.253 . 


\section{Resultados y discusión}

Sobre la educación ambiental como herramienta de gestión ambiental preventiva

Dentro de la literatura que enfatiza la mirada en la cuestión ambiental, una buena parte entiende que se trata de una crisis civilizatoria (Toledo, 1990), en tanto que el modelo de producción y consumo erosiona y degrada los ecosistemas y explota a los seres humanos es insostenible (Programa de Naciones Unidas para el Medio Ambiente, 2003). La atención de la problemática conforma un claro desafío para la ciencia dado que requiere "enfoques integrativos» y estudios interdisciplinarios (Corbeta, 2019). La educación ambiental surgió como propuesta preventiva en los años setenta del siglo XX y desde entonces transita un proceso de conformación y de incorporación en los sistemas educativos (González, 2007).

La importancia de una mirada integral en materia ambiental se observa en la reflexión sobre los instrumentos de gestión que procuran la concientización y el desarrollo de prácticas de respeto por el ambiente. En este sentido, la literatura retoma, principalmente, la evaluación de impacto ambiental de las actividades que se proponen desplegar en el territorio, la aplicación de normas voluntarias y certificación ambiental y la sanción de normativas vinculadas a los recursos naturales (Minaverry y Cáceres, 2016). En menor medida, en la bibliografía se enfatiza en la consideración de la educación ambiental como instrumento de gestión ambiental de tipo preventivo con un claro potencial transformador de pautas, hábitos y de promoción de una mirada crítica respecto a la crisis ambiental que incide en la sostenibilidad del planeta. La educación ambiental se pone en marcha con el objetivo de cambiar valores y actitudes en relación con la naturaleza y, de esta forma, contribuir a una mejor comprensión sobre la sustentabilidad ambiental (Guimarães, 2000: 17).

Luego de la Conferencia de Estocolmo de 1972 se creó el Programa de Naciones Unidas para el Medio Ambiente (PNUMA) como un coordinador a nivel internacional de las acciones ligadas a la protección del entorno. A fines de la década del ochenta, con el Informe Brundtland elaborado por la Comisión Mundial sobre el Medio Ambiente y el Desarrollo, creada en 1983 por la Asamblea de la ONU, y en la década siguiente con la Conferencia de Río de 1992, se consolidó el debate ambiental en la agenda internacional. Puntualmente, se instaló la noción de desarrollo sustentable definido como «el desarrollo que satisface las necesidades de la generación presente sin comprometer la capacidad de las generaciones futuras para satisfacer sus propias necesidades» y vinculado al desarrollo económico, social y la protección del medio ambiente (ONU, 1988). ${ }^{3}$

3. En paralelo se llevaron adelante distintos eventos relacionados a la educación ambiental, entre 
Primeramente, se hizo referencia a la educación para el desarrollo sustentable en la arena educativa de la mano de la Unesco, tras la Conferencia Mundial sobre Educación y Comunicación sobre Ambiente y Desarrollo de 1992, y luego fue reemplazada por la noción de educación ambiental que adquirió mayor presencia en los currículums educativos. La Agenda 21, que tuvo lugar tras la Conferencia de Río, planteó la importancia de la educación ambiental en la promoción del desarrollo sustentable y, en su artículo 36, destacó que la educación es «fundamental para adquirir conciencia, valores y actitudes, técnicas y comportamientos ecológicos y éticos en consonancia con el desarrollo sustentable, y que favorezcan la participación pública efectiva en el proceso de toma de decisiones» (ONU, 1992).

Tras la siguiente Cumbre Mundial sobre el Desarrollo Sostenible de Johannesburgo de 2002, se firmó el Plan de Acción y la Declaración Política que planteó, entre otras cosas, que la educación es fundamental para lograr el desarrollo sostenible, ya entendido como «el proceso mediante el cual se satisfacen las necesidades económicas, sociales, de diversidad cultural y de un medio ambiente sano de la actual generación, sin poner en riesgo la satisfacción de estas a las generaciones futuras» (ONU, 2002). Se afianzó, en ese marco, la importancia de la educación ambiental.

González Gaudiano (2007) caracteriza las trayectorias que ha tenido la educación ambiental en América Latina y destaca que las nociones ligadas a la educación para el desarrollo sustentable no tuvieron el mismo arraigo en la región latinoamericana, inicialmente. Cabe señalar que las relaciones entre educación y ambiente no son nuevas, sino que «la novedad que aporta la educación ambiental es que el medio ambiente, además de medio educativo, contenido a estudiar o recurso didáctico, aparece con entidad suficiente como para constituirse en finalidad y objeto de la educación» (Alcaraz González, 2013: 83).

Así en los diversos encuentros que se han dedicado a la educación ambiental a nivel internacional se distinguen algunas orientaciones: considerar la educación ambiental como un proceso no acotado a las escuelas, incluir los distintos componentes del ambiente natural como el creado por los seres humanos y sostener un enfoque interdisciplinario (Fuentes Amaya, 2003). Entre las referencias críticas que se ha realizado a la educación ambiental en distintos eventos, se destaca su falta de descontextualización y, en este sentido, la falta de conexión a la realidad del entorno local; el énfasis en transmitir conocimientos y no en la formación o promoción de «comportamientos responsables»; el énfasis en la conservación y protección de la vida silvestre, «descuidando dimensiones económicas y socioculturales» (Condenanza y Cordero, 2013:49).

ellos, el Coloquio Internacional sobre la Educación relativa al Medio Ambiente (Belgrado, 1975), la Conferencia Intergubernamental sobre Educación Ambiental, organizada por la Unesco y el PNUMA en Tiflis (antigua URSS, 1977), y el Congreso sobre Educación y Formación Ambiental (Moscú, 1987). Véase Alcaraz González (2013). 
Más allá de las diversas definiciones, y entendiendo que no se trata de un listado de propuestas y recetas únicas, Bachmann (2008) señala que se destacan aspectos comunes en la educación ambiental, como la referencia a la conciencia crítica acerca de la importancia del ambiente en la formación ciudadana, la formación de valores y comportamientos que promuevan la preservación del ambiente y la investigación que persiga soluciones a los desafíos ambientales. Aunque ciertamente, desde la educación ambiental se promueve la responsabilidad y participación en el tratamiento de la crisis y los problemas ambientales, hay que reconocer que no toda la población y los actores sociales y económicos tienen el mismo grado de responsabilidad. Como sostiene García (2002: 5), en muchas ocasiones, «los gestores y los técnicos evitan su responsabilidad cuando se dan consecuencias indeseadas, diluyéndose en una responsabilidad dispersa y difusa, en la que todos somos culpables y nadie, por tanto, es culpable en concreto». La atomización de las responsabilidades tiende a obviar la responsabilidad propia del sistema económico predominante, altamente ineficiente en términos ambientales y sociales.

Los principales antecedentes en la República Argentina sobre la temática incluyen algunas investigaciones que analizan desde una mirada histórica la génesis de los debates sobre la incorporación de la educación ambiental en los años ochenta, los avances de la materia en los programas y otros sobre la transversalidad de los conceptos. ${ }^{4}$

Ciertamente, la educación ambiental como motor de cambios no se vincula únicamente con la educación formal, sino con la comunidad en general (Colom y Sureda, 1999), y sobre la cual proyecta transformaciones en los «valores y sensibilidades orientados a las actividades humanas en relación con el ambiente, originando una conciencia crítica para analizar los procesos socioambientales» (Avendaño, 2012: 13). En este sentido, la educación ambiental tiene el potencial de contribuir a evitar o reducir los daños sobre el planeta, y su desconocimiento, sin duda, puede agravar la crisis ecológica (Munévar Quintero, 2013: 51). Tal como sostiene Leff (2004: 2), «la crisis ambiental es la primera crisis del mundo globalizado producida por el desconocimiento del conocimiento».

A su vez, la educación ambiental se enlaza con el acceso a la información pública ambiental que contribuye al derecho humano a un ambiente sano. Como sostiene Cáceres Colombo (2020: 1), la información «resulta imprescindible para una efectiva tutela de este derecho fundamental». La dimensión jurídica de la educación ambiental aporta información, contribuye a la promoción de conciencia crítica y a la generación de «mecanismos e instrumentos jurídicos que la forman y le permiten aplicar estrategias de acción a través de la participación» (Munévar Quintero, 2013: 61). A continuación, se caracteriza el marco normativo que reconoce en el país la educación ambiental.

4. Se destacan los trabajos de Condenanza (2014), Condenanza y Cordero (2013), Giuffré, Formento y Ratt (2007) y Corbeta (2019). 


\section{El ordenamiento jurídico de la educación ambiental}

\section{La legislación nacional sobre educación ambiental}

El ordenamiento jurídico de la República Argentina reconoce un sistema institucional de carácter federal en el que se encuentran distintas leyes y regulaciones sobre educación ambiental. Cabe señalar que el federalismo se organiza política y administrativamente en, al menos, dos ámbitos de gobierno, cada uno con una determinada distribución de atribuciones y funciones (Guillén López, 1999). Estos ámbitos refieren a una división territorial del poder y la autoridad entre el nivel federal o subnacional y el central, y que lo torna complejo y conflictivo por las distintas atribuciones y funciones que corresponden a cada nivel. El federalismo está asociado a un entramado institucional y de relaciones complejo de articular. En materia ambiental, en el país hay una distribución de funciones y competencias entre el Estado nacional, y los niveles provincial y local. Por otro lado, la educación está descentralizada (salvo la educación universitaria) y conforma una responsabilidad de las provincias. Por esta razón, la educación ambiental en el país requiere considerar dos niveles de formulación de políticas: el nacional, que se considerará en esta sección, y el provincial, al que dedicaremos la la siguiente.

En materia ambiental, la reforma de la Constitución Nacional de 1994 incorporó el derecho a un ambiente sano, equilibrado, apto para el desarrollo humano, cuyas características son la concepción derecho-deber de un ambiente sano en cuanto a su exigibilidad y participación; el compromiso intergeneracional de preservación del ambiente para las generaciones presentes y futuras; la incorporación de la noción de desarrollo sustentable, apto para el desarrollo humano; la jerarquía constitucional de la obligación de recomponer el daño ambiental y que estableció que las autoridades tienen que proteger el derecho, el acceso a la información y a la educación ambiental (artículo 41). Por esto, la educación ambiental tiene mandato constitucional en la República Argentina de forma explícita. En otros países de la región de organización federal, como México, la educación ambiental no se visibiliza tan claramente en el articulado de la Constitución y, en todo caso, se puede desentrañar en un sentido amplio la interrelación entre el derecho a recibir educación, a un medio ambiente sano y el respeto a los propios derechos humanos (Álvarez de Jesús, 2017: 114). En el caso de Brasil hay un reconocimiento constitucional de la promoción de la educación ambiental (inciso cuarto del artículo 4 Constitución de Brasil).

La incorporación del tema ambiental estuvo ligada, también, a la firma de acuerdos con organismos internacionales de financiamiento, como el Banco Interamericano de Desarrollo. Ya en 1994, durante el gobierno del presidente Carlos Menem (1989-1999), se acordó la creación del Programa de Desarrollo Institucional Ambiental, cuya función apuntó a promover el fortalecimiento legal e institucional relativo a 
la gestión ambiental. Este programa, ejecutado por la entonces Secretaría de Recursos Naturales y Desarrollo Sustentable, se extendió entre 1994 y 1999 y apuntó a jerarquizar la cuestión ambiental y su inserción programática en la estructura del propio Estado (Espinoza, 2005). Uno de los subprogramas que llevó adelante se denominó de apoyo al establecimiento de un sistema nacional ambiental y dentro de sus componentes ubicó a la educación ambiental (junto con la política y legislación ambiental, el fortalecimiento institucional y de los sistemas de información ambiental). La Resolución 1346 de 1997 de la Secretaría de Recursos Naturales y Desarrollo Sustentable cristalizó la estrategia ambiental, aunque su incidencia fue reducida aun cuando se ejecutaron las actividades previstas: «La institucionalidad ambiental todavía se caracterizaba por la existencia de múltiples órganos con competencia ambiental tanto a nivel nacional, provincial y comunal, relativamente desarticulados y que limitaban el desarrollo de una gestión ambiental eficiente» (Espinoza, 2005: 46). Esta situación de fondo no tuvo mayores modificaciones.

Posteriormente, y ya entrado el siglo XXI, tras reiterados cambios en la estructura de la dependencia responsable de la política ambiental en el país, en un marco de crisis social, política, económica, e institucional se sancionó un conjunto de normativas ambientales en carácter de presupuestos mínimos. ${ }^{5}$ Así, durante el gobierno provisional del presidente Eduardo Duhalde (2002-2003) se sancionó la Ley 25.675 General del Ambiente que, en su artículo 2, estableció que la política ambiental en el país deberá «promover cambios en los valores y conductas sociales que posibiliten el desarrollo sustentable, a través de una educación ambiental, tanto en el sistema formal como en el no formal», y en este sentido entendió a la educación ambiental como instrumento de política y de gestión ambiental. La ley señaló que la educación ambiental es un «instrumento básico para generar en los ciudadanos, valores, comportamientos y actitudes que sean acordes con un ambiente equilibrado, propendan a la preservación de los recursos naturales y su utilización sostenible, y mejoren la calidad de vida de la población» (artículo 14). Así también planteó que la educación ambiental «constituirá un proceso continuo y permanente, sometido a constante actualización que, como resultado de la orientación y articulación de las diversas disciplinas y experiencias educativas, deberá facilitar la percepción integral del ambiente y el desarrollo de una conciencia ambiental» (artículo 15).

Además, se acordó que las autoridades competentes coordinarán acciones en un ámbito federal denominado Cofema para implementación de los planes y programas de educación ambiental. Cabe señalar que el Cofema se creó a partir del denominado Pacto Federal Ambiental de 1993, como un sujeto de derecho público conformado por las provincias, la ciudad Autónoma de Buenos Aires y el Estado. El Consejo tiene

5. En Gutiérrez e Isuani (2014) se presenta un estudio del proceso de institucionalización del tema ambiental en la República Argentina. 
dentro de sus objetivos «propiciar programas y acciones de educación ambiental, tanto en el sistema educativo formal como en el informal, tendientes a elevar la calidad de vida de la población» (Acta Constitutiva del Cofema). A su vez, su asamblea constituyó comisiones de trabajo que realizaron informes y funcionaron como espacio de consulta.

En la normativa educativa propiamente tal, la educación ambiental se incorporó en 1993 en la, por entonces, Ley 24.195 Federal de Educación, dentro de los contenidos transversales para el sistema educativo obligatorio. En lo que concierne al nivel superior, la Ley 24.521 de Educación Superior de 1995, que regula la actividad de las universidades e institutos universitarios, estatales o privados y los institutos de educación superior de cada jurisdicción del país, plantea que entre los fines de la educación superior se encuentra "consolidar el respeto al medio ambiente». Es la única referencia al tema ambiental en la normativa del nivel superior.

Recién en 2006, durante el gobierno del presidente Néstor Kirchner (2003-2007), tras la sanción de la nueva Ley 26.206 de Educación Nacional que rige actualmente para la educación obligatoria (el nivel superior no lo es), tomó cierto impulso a nivel nacional la educación ambiental. Esta ley modificó la estructura general del sistema educativo, estableció cambios importantes en tanto reconoció a la educación como un bien público, impuso la obligatoriedad de los estudios secundarios y dispuso que el CFE disponga las medidas necesarias para proveer la educación ambiental. El CFE constituye una instancia federal de concertación de la educación entre las distintas jurisdicciones del país.

En el artículo 89 de dicha ley estableció que el Ministerio de Educación, Ciencia y Tecnología, en el marco del CFE:

dispondrá las medidas necesarias para proveer la educación ambiental en todos los niveles y modalidades del Sistema Educativo Nacional, con la finalidad de promover valores, comportamientos y actitudes que sean acordes con un ambiente equilibrado y la protección de la diversidad biológica; que propendan a la preservación de los recursos naturales y a su utilización sostenible y que mejoren la calidad de vida de la población.

Así también instituyó que en el CFE se coordinarán «las políticas y estrategias destinadas a incluir la educación ambiental en los contenidos curriculares comunes y núcleos de aprendizaje prioritario, así como a capacitar a los/as docentes en esta temática» (artículo 89). Hasta aquí queda en evidencia que a nivel nacional la educación ambiental conforma una responsabilidad de dos espacios de concertación: el Cofema y el CFE. ${ }^{6}$

6. En materia educativa, cabe señalar que en el CFE se establecieron los contenidos comunes de las distintas áreas de conocimiento que se consideraron significativas enseñar. Esto apunta a garantizar la 
Ya en 2013, durante el segundo gobierno de Cristina Fernández (2011-2015), en base a lo establecido en la Constitución Nacional, la Ley General del Ambiente y la Ley Nacional de Educación, se aprobó un Acuerdo Federal Educación-Ambiente implementados por las dos instancias de coordinación ya mencionadas. Dicho acuerdo entiende a la educación ambiental como «un proceso continuo y planificado cuya finalidad es la construcción de valores, conocimientos, aptitudes y actitudes orientadas no solo a la prevención» de problemas, sino a la participación crítica y reflexiva sobre el modelo desarrollo económico y social sustentable que respete «la integridad cultural, los procesos ecológicos esenciales, la diversidad biológica y los sistemas que sostienen la vida» (Resolución 260 de 2013). Además, plantea que la educación ambiental puede contribuir a generar un ámbito propicio para desarrollar nuevas actitudes basadas en valores y enfatiza en la importancia de considerar las distintas escalas - global, nacional, regional y local-y los factores sociales, económicos, políticos y culturales para afrontar la crisis ecológica, así como la integración transversal de los problemas ambientales, la participación comunitaria, la sanción de una ley con financiamiento para acciones de educación ambiental, la incorporación de la mirada de género en la temática ambiental y asegurar el acceso a la información ambiental. El acuerdo propuso la definición de los lineamientos epistemológicos y metodológicos para la educación ambiental, como eje transversal en los currículums educativos. Esta se suma al planteamiento de contenidos ambientales obligatorios (y mínimos) que se implementaron en el marco de un instrumento denominado Núcleos de aprendizaje prioritario. Estos núcleos conforman un listado de contenidos curriculares de las distintas áreas de conocimiento que se consideraron relevantes, centrales y significativos en la enseñanza obligatoria y que cada provincia tiene que incluir en sus planes de estudio.

Un año después, en 2014, se retomó la importancia de «articular las políticas de educación y las políticas ambientales, para la formación de una ciudadanía informada, participativa y ambientalmente responsable» (Resolución 283 de 2014). Esto tuvo lugar en un marco de mayor visibilización nacional de los conflictos ambientales en el país, vinculados, fundamentalmente, con la industria papelera, minera y a situaciones de notoria contaminación hídrica que afectan a diversas cuencas. Esta situación derivó en un proceso de creciente judicialización en materia ambiental, aunque no se avanzó en la profundización de acciones en materia de educación ambiental y estas se mantuvieron dispersas y atomizadas.

Un elemento que se destacó en los siguientes años se vincula a diversos intentos por sancionar una ley de educación ambiental específica reconociendo la baja implementación que tiene en las jurisdicciones provinciales. Durante 2007, 2011 y 2015 se

integración y la validez nacional de los títulos, en un país que tiene veintitrés provincias, además de la ciudad autónoma de Buenos Aires. 
presentaron diversos proyectos de ley sobre educación ambiental que fueron tratados en las comisiones de Educación y de Recursos Naturales y de Conservación del Ambiente Humano, con aprobación de la Cámara de Diputados de la Nación, pero no fueron tratados en la Cámara de Senadores de la Nación. Estos proyectos buscaron acordar una estrategia nacional para orientar y articular las acciones en el territorio, que declaraba como de interés público y prioridad nacional, considerando su importancia en la preservación del ambiente, el mejoramiento de la calidad de vida y el desarrollo sustentable, rescatando la dimensión ética de la sustentabilidad, entre otros.7

En 2017, durante el gobierno de Mauricio Macri (2015-2019), el CFE y el Cofema aprobaron un nuevo compromiso federal destinado a promover las políticas públicas de educación ambiental para el desarrollo sustentable. En ese mismo año, mediante la Resolución 343 de 2017, se conformó dentro del propio Cofema la Comisión de Educación Ambiental. Además, se declaró de interés federal un proyecto de ley de educación ambiental, elaborado con participación de la Secretaría de Ambiente y Desarrollo Sustentable, y analizado en el Cofema. Dicho proyecto de ley se presentó en el Congreso de la Nación en 2018 por diputados del oficialismo, y en sus fundamentos se «reconoce la centralidad pública de la cuestión ambiental, la complejidad del ambiente y el valor estratégico y ético de la educación ambiental». ${ }^{8}$ El proyecto defendió el derecho de los y las estudiantes a recibir educación ambiental con el propósito de promover el desarrollo sustentable, las prácticas ciudadanas responsables en materia ambiental, la conciencia del cuidado del ambiente, etcétera.

Además del proyecto del oficialismo, en 2018 también se presentó otro proyecto de ley por parte de legisladores del bloque opositor que declaraba de interés público y prioridad nacional la estrategia nacional de educación ambiental, tendiente a propiciar «la construcción de visiones críticas y concepciones diversas respecto del modelo de relación que la sociedad moderna establece con la naturaleza; favorecer el reconocimiento social de la complejidad de los conflictos ambientales, la comprensión de su multicausalidad y la interdependencia entre factores», entre otros. ${ }^{9}$

Los proyectos presentados por diputados del oficialismo y por diputados opositores fueron tratados juntos a fines de 2019, antes del recambio de gobierno, en la Cámara de Diputados de la Nación. Sin embargo, no llegaron a tratados por la Cámara de Senadores de la Nación, es decir, no se convirtieron en ley.

En paralelo, en la Cámara de Senadores de la Nación distintos bloques también prese $^{10}$ ntaron proyectos sobre educación ambiental: el Expediente 2437/18 propuso

7. Expedientes 279-D.-200, 2.771-D.-2006, 1.006-D.-2014, 1.314-D.-2014, 1.982-D.-2014, 2.090-D.2014, 2.201-D.-2015.

8. Cámara de Diputados de la Nación, Expediente 6230-D-2018.

9. Cámara de Diputados de la Nación, Expediente 6755-D-2018.

10. Expedientes 3220/15, 149/15, 2086/17, 2486/19. 
establecer presupuestos mínimos de educación ambiental, y la creación de un Consejo Nacional de Ambiente y Educación como espacio de «confluencia, concertación y formulación de la política de educación ambiental nacional». Además, en otros proyectos se impulsaba una estrategia nacional como política concertada y permanente que incorporaba la educación ambiental como materia específica dentro de la educación primaria y secundaria y la intensificación de campañas de concientización, entre otros. Llamativamente, algunos de estos proyectos ignoraban que ya existían contenidos mínimos respecto a la protección del ambiente en la educación obligatoria. Ninguno de los proyectos se convirtió en ley.

Nuevamente en 2020, durante el gobierno del presidente Alberto Fernández, se presentó nuevamente en la Cámara de Diputados un proyecto de ley sobre educación ambiental por parte de diputados del oficialismo (Expediente 2967-D-2020), que retoma estos debates y que aún no ha tenido avances. Poco después, el presidente, su jefe de gabinete y los ministros de Ambiente y Educación enviaron el Mensaje 0130/20 y el proyecto de ley del Expediente oo10-PE-2020 que retoma estos debates. Finalmente, tras su tratamiento por las comisiones específicas, tuvo media sanción de la Cámara de Diputados de la Nación. Posteriormente, en mayo de 2021, en plena pandemia del covid-19, la Cámara de Senadores sancionó la Ley 27.621 para la Implementación de la Educación Ambiental Integral, que se publicó en el Boletín Oficial en junio mediante el Decreto 356 de 2021.

La Ley 27.621 sostiene la educación ambiental como un proceso "permanente, integral y transversal» y retoma algunos principios de otras normas, como la Ley General del Ambiente. Así, se fundamenta en los principios del abordaje interpretativo y holístico que reconoce la interdependencia de los distintos elementos del ambiente; el respeto y valor por la diversidad; la equidad, la igualdad desde una mirada de género con el reconocimiento teórico de las corrientes del ecofeminismo; el reconocimiento de la diversidad cultural, la formación ciudadana, el cuidado del patrimonio natural y cultural, la problemática ambiental y los procesos sociohistóricos, la educación en valores, el pensamiento crítico y el ejercicio del derecho al ambiente sano (artículo 3).

Una novedad es la incorporación de la temática de género en el abordaje de la cuestión ambiental que permite profundizar el debate y trazar relaciones. El objetivo central es la implementación de una Estrategia Nacional de Educación Ambiental Integral y en este sentido tendrá que fortalecer las capacidades técnicas mediante la profesionalización de los recursos humanos y generar mecanismos que contribuyan al logro de la Agenda 2030 con sus Objetivos de Desarrollo Sostenible (artículo 4). También esta norma reconoce la importancia que tiene la formulación coordinada de Estrategias Jurisdiccionales de Educación Ambiental Integral y acuerda la educación ambiental en tres ámbitos formales (el sistema educativo obligatorio y universidades), el no formal (con las organizaciones de la sociedad civil) y el informal (con los medios de comunicación). 
Como novedad institucional creó el Consejo Consultivo de la Estrategia Nacional de Educación Ambiental Integral que tendrá la tarea «de asistir y asesorar» a las autoridades en la implementación de la estrategia nacional (artículo 14). Por último, incorporó el inciso g) a la Ley 26.206 de Educación, que en su artículo 92 sobre contenidos curriculares, sostiene que «la toma de conciencia de la importancia del ambiente, la biodiversidad y los recursos naturales, su respeto, conservación, preservación y prevención de los daños», en concordancia con el resto de la normativa ambiental.

En paralelo a estas instancias, en los últimos años, el Ministerio de Ambiente y Desarrollo Sustentable mantuvo algunas iniciativas de educación ambiental. Entre ellas, organizó dos encuentros nacionales de educación ambiental con representantes de provincias, donde se discutió la necesidad de ejecutar políticas públicas, la asignación presupuestaria específica, la articulación intersectorial, la planificación regional de programas y la necesidad de contar con materiales didácticos y de divulgación que consideren las particularidades regionales, entre otros (Secretaría de Ambiente y Desarrollo Sustentable, 2019). Estas actividades contaron con la participación de las jurisdicciones, pero no se realizaron en el marco del Cofema ni del CFE, organismos de coordinación de las decisiones. De hecho, en las resoluciones que emiten estas instancias de concertación no se hace referencia a los encuentros.

Por lo señalado, la educación ambiental está establecida en la Constitución Nacional, en la Ley General del Ambiente y en la Ley de Educación y, recientemente, en la Ley de Presupuestos Mínimos para todo el país (tabla 1 ).

Tabla 1. Normativas nacionales de educación ambiental

\begin{tabular}{|c|c|c|}
\hline Norma & Año & Contenido \\
\hline Constitución de la Nación & 1994 & $\begin{array}{l}\text { Artículo } 41 \text { establece que las autoridades son responsables de proveer educación } \\
\text { ambiental }\end{array}$ \\
\hline Ley 25.675 General del Ambiente & 2002 & $\begin{array}{l}\text { Se entiende a la educación ambiental como proceso continuo y permanente; y } \\
\text { como instrumento de política y de gestión ambiental tendiente a generar cambios } \\
\text { de valores, comportamientos, etc. }\end{array}$ \\
\hline Ley 26.206 de Educación Nacional & 2006 & $\begin{array}{l}\text { Responsabilidad de proveer educación ambiental en todos los niveles del sistema } \\
\text { educativo tendiente a promover valores, comportamientos y actitudes que protejan } \\
\text { la diversidad, los recursos naturales, y calidad de vida de la población }\end{array}$ \\
\hline $\begin{array}{l}\text { Ley } 27.621 \text { de Educación Ambien- } \\
\text { tal Integral }\end{array}$ & 2021 & $\begin{array}{l}\text { Se entiende a la educación ambientalcomo un proceso «permanente, integral y } \\
\text { transversal» desde una mirada que reconoce la interdependencia de los elementos } \\
\text { del ambiente. Destaca avanzar en un compromiso ambiental intergeneracional. } \\
\text { Incorpora las perspectivas ecofeministas y crea un consejo consultivo. }\end{array}$ \\
\hline
\end{tabular}

Fuente: Elaboración propia 


\section{La legislación subnacional sobre educación ambiental}

En la sección anterior se caracterizó la situación normativa e institucional a nivel nacional, aunque cabe explicar que la educación ambiental está contemplada —en algunos casos en forma previa a la sanción de la Ley Nacional de Educación y de la reciente Ley de Presupuesto Mínimo- en una profusa legislación subnacional bajo cuatro modalidades: a) como una ley específica; b) como un artículo dentro de normativa educativa; c) como un artículo dentro de normativa ambiental; y d) como un artículo en normativas educativas y ambientales.

En el primer caso se encuentran jurisdicciones diversas, como la provincia de Chaco, que tiene la Ley 1114-E desde 2001, la Ciudad Autónoma de Buenos Aires que sancionó la Ley 1.687 en 2005, Misiones con la Ley XVI 80 en 2005, Río Negro con la Ley 4.242 de 2007, Chubut con el Decreto 350 de 2012, Entre Ríos con la Ley 10.402 de 2014 y Catamarca con la Ley 5.424 de 2014. Estas normativas específicas tienen como objetivos el desarrollo de la conciencia ambiental, la mirada crítica y promoción de prácticas favorables con el ambiente, la participación de la comunidad, la mirada crítica de la realidad socioambiental local, la incorporación transversal en los diseños curriculares, la promoción del desarrollo sustentable y la construcción de saberes ambientales, y determinan autoridades de aplicación.

En el segundo caso se encuentran la provincia de Buenos Aires con la Ley de Educación 13.688 de 2007, que dentro del artículo 45 incluyó la educación ambiental; la provincia de Santa Cruz con la Ley 3.305 de 2012 en el artículo 145; y Neuquén, con la Ley 2.945 de 2014, que en su artículo 21 establece contenidos curriculares transversales y obligatorios. Entre los aspectos que consideran estos artículos se encuentran la relevancia de aportar propuestas curriculares y extracurriculares para la incorporación de la perspectiva ambiental, su presencia como contenido transversal, la formación y capacitación para los docentes, la promoción de prácticas permanentes de gestión ambiental y valores y comportamientos acordes con un ambiente equilibrado y la protección de la diversidad biológica.

En el tercer caso se diferencian aquellas provincias que contemplan la educación ambiental en el marco de una ley de ambiente. En dicha situación se encuentran las provincias de Tucumán, que tiene la Ley 6.253 sobre normas generales y metodología de aplicación para la defensa, conservación y mejoramiento del ambiente de 1991 en sus artículos 3, 4 y 16; la provincia de Tierra del Fuego con la Ley Provincial Medio Ambiente 55 de 1992, en sus artículos 20, 21 y 22 y 23; la provincia de Formosa, con la Ley 1.06o de Política Ecológica y Ambiental de 1993, en sus artículos 9, 127 y 128; la provincia de Salta, con la Ley de Protección del Ambiente 7.070 del 2000, en su artículo 5; la provincia de La Rioja, con la Ley 7.801 de 2005, en sus artículos 87 al 91; la provincia de San Luis, con la Ley I-0648 de 2008, en sus artículos 15 y 23; Santa Fe 
con la Ley Provincial 11.717 de Medio Ambiente y Desarrollo Sustentable de 2009, en su artículos 2 y 15; Córdoba con la Ley de Política Ambiental 10.208 de 2014, en su artículo 8; Corrientes, con la Ley 6.265 del 2014, que estableció la semana de concientización del ambiente; y, en Jujuy, con la Ley 5.063 de 2017, en sus artículos 58 y 59. Estas normativas consideran a la educación ambiental como un instrumento o herramienta de gestión y política ambiental tendientes a avanzar en el desarrollo sustentable, la obligatoriedad de la incorporación en los currículums y su interdisciplinariedad, entre otros aspectos relevantes.

Por último, en el cuarto caso se encuentran las provincias que incluyeron la educación ambiental dentro de las normativas educativas y ambientales. En esta situación se encuentra la provincia de Mendoza que tiene, por un lado, la Ley 5961 de 1992 de preservación del medio ambiente, específicamente en sus artículos 43 y 44 donde incorpora la educación ambiental en los programas de estudio; y por otro, el artículo 20 de la Ley de Educación Provincial 6970 de 2002. Así también la provincia de Santiago del Estero que tiene la Ley de Educación 6.876 de 2007, que a través de su artículo 106 incorpora la temática ambiental en todos los niveles y modalidades del sistema educativo, con la finalidad de promover valores relacionados a un ambiente equilibrado y la protección de la diversidad biológica; y tiene además la Ley 6.321 de Defensa, Conservación y Mejoramiento del Ambiente y los Recursos Naturales de 1996 en cuyos artículos 22 y 23 incorporan contenidos ecológicos, la promoción de jornadas ambientales y la conformación de espacios de participación. En similar situación la provincia de San Juan cuenta con la Ley General del Ambiente 513-L de 2014 que acuerda un plan permanente de educación y concientización de ambiental en sus artículos 10 al 13; y la Ley de Educación 1327-H de 2015 que en su artículo 226 establece a la educación ambiental como eje transversal en el sistema educativo, su incorporación en el diseño curricular, tendiente a la formación crítica y favorecer valores y compromisos ligados al desarrollo sustentable.

El recorrido presentado hasta aquí permite observar que existe una profusa legislación a nivel subnacional que retoma la educación ambiental con distintos sentidos y modalidades. Salvo algunas excepciones, como en el caso de las provincias de Mendoza, Formosa, Tucumán y Tierra del Fuego, la mayoría de las jurisdicciones tienen normativas sancionadas desde inicios del siglo XXI que se relacionan con la emergencia del tema ambiental en la agenda pública. A su vez, vinculan la educación ambiental con el derecho humano al ambiente sano y con otros derechos, aunque hay diversidad de principios, contenidos y valores.

\section{Conclusiones}

El trabajo permitió indagar el recorrido que, precisamente, dio lugar a la emergencia en la legislación de la República Argentina de la educación ambiental en el sistema 
educativo formal obligatorio. Se identificó, sistematizó y estudió la profusa normativa vigente a nivel nacional y provincial que la reconoce. El proceso permitió observar que la educación ambiental a nivel nacional está referenciada en dos espacios de coordinación federal, el Cofema y el CFE, que acuerdan parámetros para todo el país. Aunque esta normativa está altamente fragmentada, se observan los siguientes aspectos comunes: la incorporación transversal de forma obligatoria en los currículums, la consideración de la educación como herramienta de política y gestión ligada a la modificación de hábitos y la generación de conciencia crítica y la consideración del desarrollo sostenible.

En el marco de la crisis ambiental planetaria que tensiona los países de la región latinoamericana, la educación ambiental es una herramienta de gestión ambiental preventiva desde la cual es posible reflexionar críticamente en torno a los modelos productivos y a los patrones de consumo. Y, en ese marco, contribuir a la construcción de alternativas que, sin desconocer las heterogeneidades, las históricas desigualdades sociales, económicas y ambientales, contribuyan a la protección del ambiente. En este sentido, la educación ambiental se convierte en una oportunidad de "construcción de una nueva sensibilidad y visión del ambiente tanto en el ámbito educativo formal como no formal», y aporta a la "concienciación y capacitación sobre problemas ambientales locales-globales», lo cual «podría ser la llave hacia el desarrollo de valores y comportamientos diferentes en la relación de los seres humanos con el ambiente» (Martínez Castillo, 2012: 2).

La sensibilización y la toma de conciencia son aspectos presentes en distintas perspectivas de la educación ambiental, pero no pueden quedar reducidos a las «características del problema y sus consecuencias (por ejemplo, la naturaleza del efecto invernadero y su relevancia en el cambio de clima), sino también sobre los diversos intereses sociales en juego y las razones políticas»; hay que aprovechar «los pocos resquicios que deja el sistema a la crítica y a la transformación social (sentido de la resistencia y solidaridad con las personas y colectivos que comparten esta posición)» (García, 2002: 4).

En un país federal con tradiciones educativas diversas y gobiernos de distintos signo político-partidario, con la educación descentralizada en 24 jurisdicciones, la sanción de normativas que empujan la educación ambiental como un instrumento de gestión ambiental de tipo preventivo es un aspecto a destacar. En el marco de la atomización reinante y una trayectoria institucional errática y cambiante en el campo ambiental (que deviene en bajos niveles efectivos de implementación de una política ambiental), la sanción de la Ley 27.621 de Educación Ambiental Integral tiene el potencial de reflexionar sobre los contenidos de forma transversal, con principios reconocidos en la literatura (solidaridad intergeneracional, protección del patrimonio cultural, la mirada holística de los procesos ambientales, la cuestión de género, entre otras). Por delante queda pendiente el trabajo de promoción que permita consolidarla, efectivamente, en el sistema educativo. 


\section{Referencias}

Alcaraz GonzÁlez, Inmaculada (2013). Manual monitor de educación ambiental. España: CEP.

Álvarez de Jesús, José (2017). Educación ambiental en México. Análisis desde un enfoque jurídico. Tesis de la maestría en Derecho Ambiental, Universidad Autónoma del Estado de México.

Avendaño, William (2012). «La educación ambiental (educación ambiental) como herramienta de la responsabilidad social». Revista Luna Azul, 35: 94-115. Disponible en https://bit.ly/3IWSmRD.

Bachmann, Lía (2008). La educación ambiental en Argentina, hoy. Documento marco sobre Educación Ambiental. Dirección Nacional de Gestión Curricular y Formación Docente Áreas Curriculares, Ministerio de Educación, Buenos Aires. Disponible en https://bit.ly/3yB $7 \mathrm{IgL}$.

Cáceres Colombo, Nicolás (2020). «Acceso a la información y educación ambiental». Diario Ambiental, 281. Disponible en https://bit.ly/3meqcrG.

Colom, Antonio y Jaume Sureda (1999). El hombre en el medio. Orientaciones metodológicas para el desarrollo dirigido a la formación de valores, la disciplina y la responsabilidad ciudadana desde la escuela. La Habana: CEAC, MINED.

Condenanza, Lucía y Silvina Cordero (2013). «Educación ambiental y legislación educativa en Argentina. Hacia un estado de la cuestión». Praxis Educativa, 17 (1): 47-55. Disponible en https://bit.ly/3yC61sx.

Condenanza, Lucía (2014). «Aportes para historizar la educación ambiental en Argentina: La cuestión ambiental en la Ley Federal de Educación. Historia de la Educación». Memoria Académica, 15 (1): 5-28. Disponible en https://bit.ly/3mheH2N.

CoRbETA, Silvia (2019). «Educación y ambiente en la educación superior universitaria: Tendencias en clave de la perspectiva crítica latinoamericana». Revista Educación (Costa Rica), 43 (1): 1-16.

Espinoza, Guillermo (2005). Evaluación de proyectos de desarrollo institucional Un análisis comparativo. Banco Interamericano de Desarrollo Washington, D.C. Serie de informes técnicos del Departamento de Desarrollo Sostenible. Disponible en https://bit.ly/3yPZdbb.

Fuentes Amaya, Silvia (2003). «Los educadores ambientales de la UPN-Mexicali: hacia una articulación entre lo discursivo y lo psíquico para leer el proceso identificatorio». En Granja Castro, J. Miradas a lo educativo. Exploraciones en los límites (pp. 67-87). México: Editado por Plaza y Valdez.

GARCíA, José (2002). «Los problemas de la educación ambiental: ¿Es posible una educación ambiental integradora?». Revista Investigación en la Escuela, 46: 5-25. Disponible en https://bit.ly/3q987MD. 
GIUfFré, Lidia, Suasan Formento y Silvia Ratto (2007). «Transversalidad de conceptos de educación ambiental para un desarrollo sostenible presentes en la legislación argentina». Revista Ciencia del Suelo, 25 (1), 75-80. Disponible en https://bit. ly/30AOO6f.

GonzÁLez, Edgar (2007). Educación ambiental: Trayectorias, rasgos y escenarios. México: Plaza y Valdés.

Guillén López, Tonatiuh (1999). Federalismo, gobiernos locales y democracia. México: Instituto Federal Electoral.

Guimarães, Mauro (2000). Educación ambiental: no consenso um embate? Campinas-SP: Papirus.

GutiérReZ, Ricardo e Fernando Isuani (2014). «La emergencia del ambientalismo estatal y social en Argentina». Revista de Administração Pública, 48 (2): 296-322. Disponible en http://dx.doi.org/10.1590/0034-76121700.

LEFF, Enrique (2004). «Educación ambiental: Perspectivas desde el conocimiento, la ciencia, la ética, la cultura, la sustentabilidad». I Congreso Nacional de Educación Ambiental para el desarrollo sustentable de la Argentina, CTERA, Córdoba.

Minaverry, Clara y Verónica Cáceres (2016). «Fortalezas y debilidades de los instrumentos de gestión ambiental en la provincia de Buenos Aires, Argentina». Revista Mexicana de Ciencias Políticas y Sociales, 32 (56): 132-149.

Munévar Quintero, Claudia Alexandra (2013). «La incidencia de la educación jurídica ambiental y la participación, en la transformación del conflicto ambiental». Jurídicas (Manizales: Universidad de Caldas), 1(10): 147-163.

ONU (1973). Informe de la Conferencia de las Naciones Unidas sobre el Medio Humano, Estocolmo. Disponible en https://bit.ly/3p853kV.

ONU (1988). Informe de la Comisión Mundial del Medio Ambiente y del Desarrollo Santiago de Chile. Disponible en https://bit.ly/3F6Tj7z.

ONU (1992). Agenda 21. Documento de las Naciones Unidas. Disponible en https:// bit.ly/3EjgGKd.

ONU (2002). Informe de la Cumbre Mundial sobre el Desarrollo Sostenible. Disponible en https://bit.ly/3EooPCY.

Programa de Naciones Unidas para el Medio Ambiente (2003). Manifiesto por la vida. Por una ética para la sustentabilidad. México: Programa de las Naciones Unidas para el Medio Ambiente. Disponible en https://bit.ly/32ayQR3.

Secretaria de Ambiente y Desarrollo Sostenible, Argentina (2019). Estrategia Nacional de Educación Ambiental. Buenos Aires. Acceso el 21 de agosto de 2020. Disponible en https:/www.sib.gob.ar/portal/wp-content/uploads/2019/05/ ENEA-Estrategia-Nacional-de-Educaci\% $\mathrm{C}_{3} \%$ B3n-Ambiental.pdf

Toledo, Víctor (1990). «Modernidad y ecología. La nueva crisis planetaria». Revista Ecología Política, 3: 9-22. 


\section{Sobre la autora}

Verónica lucia Cáceres es doctora en Ciencias Sociales por la Universidad Nacional de General Sarmiento. Investigadora del Consejo Nacional de Investigaciones Científicas y Técnicas en el Instituto de Investigaciones Jurídicas y Sociales Ambrosio Lucas Gioja de la Facultad de Derecho de la Universidad de Buenos Aires. Investigadora y docente adjunta en el Instituto de Desarrollo Humano de la Universidad Nacional de General Sarmiento y en el Departamento de Ciencias Sociales de la Universidad Nacional de Luján. Su correo electrónico es vcaceres@campus.ungs.edu.ar. (iD https://orcid.org/0000-0002-1721-369X. 
La Revista de Derecho Ambiental, del Centro de Derecho Ambiental de la Facultad de Derecho de la Universidad de Chile, es un espacio de exposición y análisis en el plano académico del derecho ambiental. Su contenido se presenta a través de doctrina, jurisprudencia y recensiones, y aborda diversas materias relacionadas con la gestión, institucionalidad y herramientas de protección ambiental y desarrollo sustentable. Se presentan artículos de diferentes autores y autoras en los que se analizan y abordan casos y temas jurídico-ambientales de creciente interés y actualidad.

\author{
DIRECTORA \\ Valentina Durán Medina \\ EDITORES \\ Jorge Ossandón Rosales \\ y Antonio Pulgar Martínez \\ SITIO WEB \\ revistaderechoambiental.uchile.cl \\ CORREO ELECTRÓNICO \\ revistada@derecho.uchile.cl \\ LICENCIA DE ESTE ARTÍ́CULO \\ Creative Commons Atribución Compartir Igual 4.o Internacional
}

La edición de textos, el diseño editorial

y la conversión a formatos electrónicos de este artículo

estuvieron a cargo de Tipográfica

(www.tipografica.io) 